\title{
Electrical Behaviour and Spherulites Morphology of HDPE/PP Polyblends with HDPE as Base Material
}

\author{
Prakash Chandra Sahoo1, Rabiranjan Murmu1, Sarat Chandra Patra1, Chiranjit Dutta², \\ Harekrushna Sutar ${ }^{1,3^{*}}$ \\ ${ }^{1}$ Chemical Engineering Department, Indira Gandhi Institute of Technology, Sarang, India \\ ${ }^{2}$ Chemical Engineering Department, National Institute of Technology, Trichy, India \\ ${ }^{3}$ Chemical Engineering Department, Jadavpur University, Kolkata, India \\ Email: ^h.k.sutar@gmail.com
}

How to cite this paper: Sahoo, P.C., Murmu, R., Patra, S.C., Dutta, C. and Sutar, H. (2018) Electrical Behaviour and Spherulites Morphology of HDPE/PP Polyblends with HDPE as Base Material. Materials Sciences and Applications, 9, 837-843. https://doi.org/10.4236/msa.2018.910060

Received: July 29, 2018

Accepted: September 18, 2018

Published: September 21, 2018

Copyright $\odot 2018$ by authors and Scientific Research Publishing Inc. This work is licensed under the Creative Commons Attribution International License (CC BY 4.0).

http://creativecommons.org/licenses/by/4.0/

\begin{abstract}
Polymer composites of virgin high density poly ethylene (HDPE) and virgin polypropylene (PP) are prepared. PP of weight\% of 20,30 and 50 are reinforced to HDPE in the form of pellets. They are converted into raw polymer sheets using a two roll milling machine. The prepared raw sheets have undergone compression moulding to fabricate polymer sheets to study electrical properties like dielectric strength, surface resistivity and volume resistivity at atmospheric temperature and pressure. Result shows dielectric strength and volume resistivity decreases with addition of PP to HDPE, whereas surface resistivity increases. Crystal growth rate is observed using a cross polarised microscope (PLM). The microscopy results reveal, the PP crystallizes faster than HDPE and the growth rate declines for the polyblend; showing non-uniform and hazy spherulitic structure.
\end{abstract}

\section{Keywords}

Polymer Blend, Electrical Properties, Crystallization, Morphology

\section{Introduction}

During last decades polymeric containing materials attracted the attention of scientists for wide spread applications such as solar energy conversion, coatings, adhesives, lithography, light emitting diodes, sensors, laser development and many applications [1] [2]. Thermoplastics are used in various electrical applications like wire and cable as insulation and jacketing materials due to their unique combination of properties such as low temperature flexibility, excellent insulat- 
ing characteristics and resistance to moisture absorption [3]. Electrical properties of various polymer blends have been investigated by different researcher [4]-[10]. In general polymer blends are prepared by physical mixing of two or more polymers to obtain a new material with improved properties compared to the parent one. This is the most convenient method of obtaining a material rather than synthesizing a new polymer [11] [12]. The electrical conductivity studies are aimed at understanding the origin of the charge carrying species and the way in which they move through the bulk of the material. Polymers with controlled conductivity and thermal sensitivity are much desirable in various applications [13] [14] [15]. Knowledge of electrical properties of polymer blends is helpful in material study and characterization for device fabrication.

To develop a new electrical insulating material with good performance, it is important to do researches focusing on the effect of morphology on electrical properties. Polypropylene possesses good insulation performance because of its high crystallinity. However, since spherulite boundaries become weak points, the dielectric strength of polypropylene is not so much higher than high density polyethylene [16] [17]. It is reported that by blending polyethylene with polypropylene, the dielectric strength can be increased because the spherulite boundaries are reinforced [18] [19] [20]. The mechanism of increase in dielectric strength by polymer blending is still not so clear. In the present paper, effects of blending of polypropylene (PP) with high density polyethylene (HDPE) on different electrical properties, such as dielectric strength, surface resistivity and volume resistivity are examined. Crystal structures are observed through cross polarised optical microscope (PLM).

\section{Materials and Methods}

\subsection{Pickup of Polymer Raw Materials}

Polypropylene of homopolymer type with M110 grade prepared by the spheripol technology and high density polyethylene of injection moulded type with M5818 grade prepared by Mitsui Slurry CX technology is obtained from Haldia petrochemical limited, Haldia, West Bengal, India in the form of pellets. Different physical properties of the polymers are reported in Table 1 .

\subsection{Preparation of Polymer Sheets}

The collected polymer pellets are dried in a hot air oven at $60^{\circ} \mathrm{C}$ for 8 hours to remove moisture content followed by mixing of 20,30 and 50 weight\% of PP to HDPE. They are converted into polymer raw sheets using a two roll milling machine having front roll at a speed of $42 \mathrm{rpm}$ and rear roll at a speed of $37 \mathrm{rpm}$ at an operating temperature of $300^{\circ} \mathrm{C}$. The rollers are of $155 \mathrm{~mm}$ diameter and 360 $\mathrm{mm}$ length. The raw sheets are chopped and finally converted to circular disc shaped sheets having $\Phi$ of $110 \mathrm{~mm}$ (for surface and volume resistivity) and 50 $\mathrm{mm}$ (for dielectric strength) with $3 \mathrm{~mm}$ thickness using a laboratory compression press (Identification No.: CIPET/PTC/119, Make: CIPET, Ahmedabad) as 
per ASTM-D 1894 standard at $300^{\circ} \mathrm{C}$ and 15 tons load. The circular disc shaped polymer sheets used in the experiment are shown in Figure 1.

\section{Results and Discussion}

Electrical properties like surface resistivity and volume resistivity at atmospheric temperature and pressure are measured using a super mega ohmmeter (Identification No.: CIPET/PTC/095, Make: Toa Electronics Ltd., Japan, Model: SM-8220) at a voltage of $500 \mathrm{~V}$ as per ASTM-D 257. Dielectric strength is measured using a dielectric breakdown tester (Identification No.: CIPET/PTC/150, Megger, OTS $100 \mathrm{AF} / 2$ ). Results of different electrical properties are reported in Figures 2-4.

Since the surface length is fixed, the measurement of surface resistivity is independent of physical dimensions. In our observation the surface resistivity of both HDPE and PP samples are same. But the augmentation of PP with HDPE matrix increases the surface resistivity. The value is maximum for 70HDPE/30PP composite. The mechanism behind the improvement of the property is not understood in our project. Data pertaining to volume resistivity is shown in Figure 3. PP bears maximum volume resistivity of about $10^{16}$ Ohm.cm. It is noteworthy to mention that the reinforcement of PP to HDPE matrix results in decrease of volume resistivity. The obtained results reveal, the $80 \mathrm{HDPE} / 20 \mathrm{PP}$ composite possesses the minimum volume resistivity of $2.07 \times$ $10^{14} \mathrm{Ohm} . \mathrm{cm}$. But the causes behind the fall in the said property are unknown.

Dielectric strength is a major electrical property of insulators. In general the electrical properties break down after continuous application of increased voltage to an insulator. So it is a measure of electrical strength of a material as an insulator. It is the maximum voltage required to produce a dielectric breakdown through the material and is expressed as volts per unit thickness. In our observation the HDPE/PP composite bears less dielectric strength than their virgin forms as shown in Figure 4. Generally the dielectric strength of a material arises due to the polarisation of molecules and it increases with increase in polarisability [21]. It is believed that the augmentation PP to HDPE matrix decreases the atomic polarisation, thereby lessening the insulating properties.

Spherulite morphology is observed at magnification $\times 10$ to understand the crystal behaviour of the polymers. Cross polarised light microscope (PLM, Leica, DM 750P, Germany) is implemented for this characterisation. At incipient the heating stage of the PLM is switched on at a heating rate of $10^{\circ} \mathrm{C} /$ minute till $200^{\circ} \mathrm{C}$ is reached. The temperature is maintained constant and a tiny polymer sample of around $10 \mathrm{mg}$ is melted using the hot stage. The molten polymer is converted to a thin film placing a micro glass slide over it. Cooling stage is switched on at a rate of $10^{\circ} \mathrm{C} /$ minute and spherulite images are captured at $130^{\circ} \mathrm{C}$ and $120^{\circ} \mathrm{C}$ respectively as shown in Figure 5. The results reveal; reinforcement of PP to HDPE matrix declines the crystal growth. Growth rate is higher in PP. The spherulites bloom in a spherical shape with a uniform struc- 
ture. But the crystal structure for HDPE is like ring shaped. The polymer blend possesses both the phases having distinguishable spherulites. This may be due to the non compatibility of PP with HDPE matrix.

Table 1. Physical properties of the collected polymers.

\begin{tabular}{ccc}
\hline Polymer type & Melt flow index $(\mathrm{g} / 10 \mathrm{~min})$ & Density $(\mathrm{g} / \mathrm{cc})$ \\
\hline HDPE & $19\left(2.16 \mathrm{~kg}, 190^{\circ} \mathrm{C}\right)$ & 0.956 \\
PP & $11\left(2.16 \mathrm{~kg}, 230^{\circ} \mathrm{C}\right)$ & 0.900
\end{tabular}

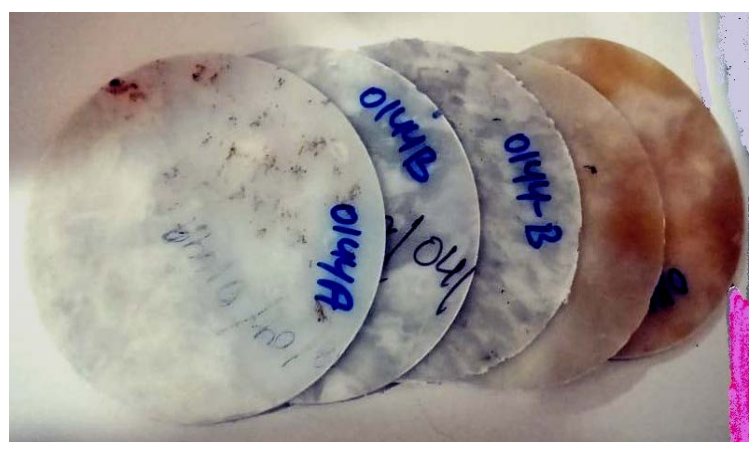

Figure 1. Polymer sheets incorporated for electrical property study.

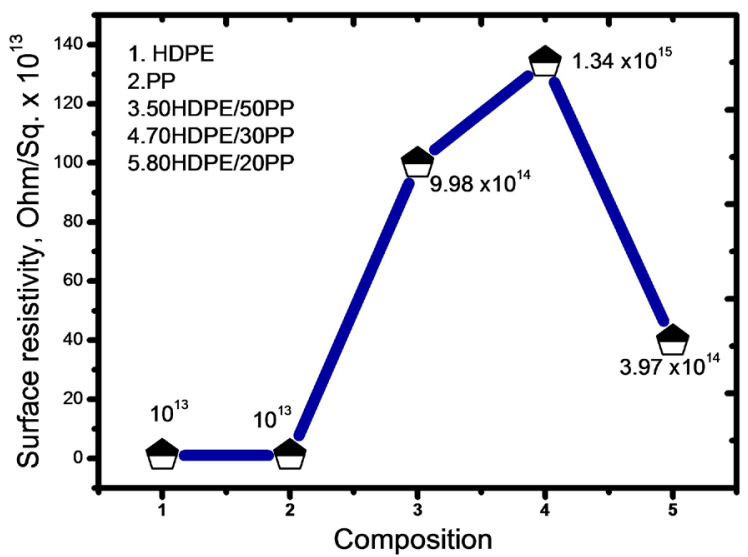

Figure 2. Results showing data for surface resistivity.

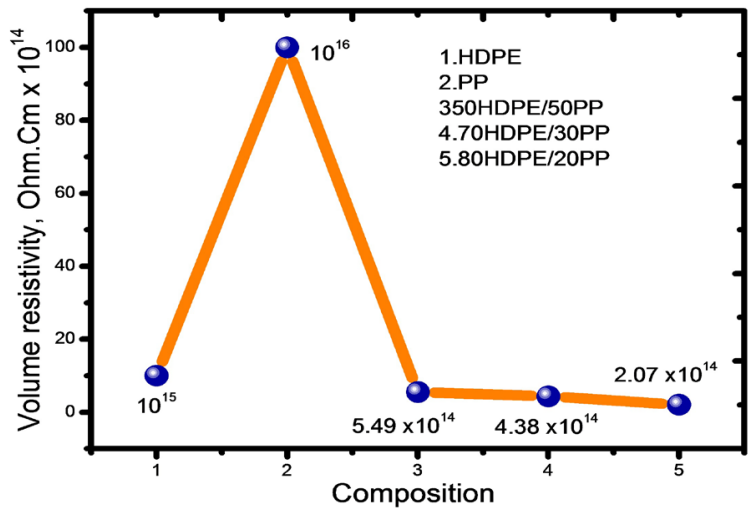

Figure 3. Variation of Volume resistivity with composition. 


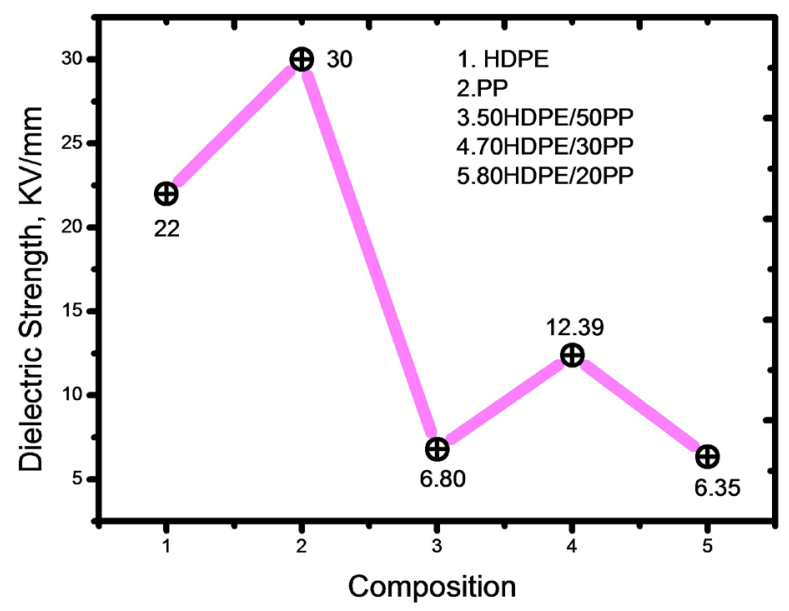

Figure 4. Dielectric Strength as a function of composition.

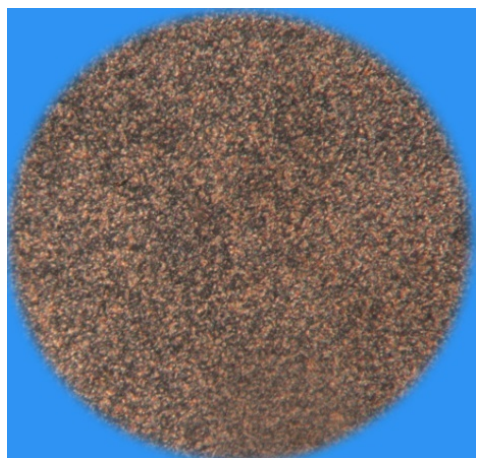

(a)

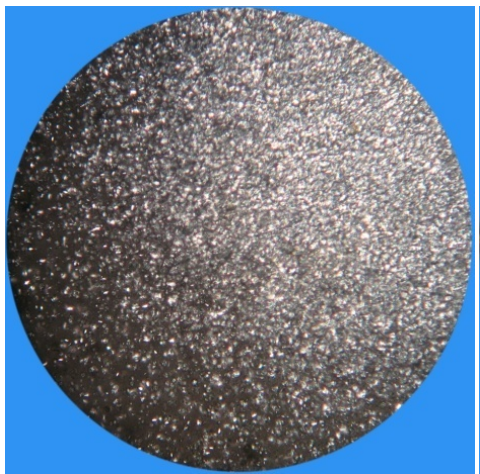

(c)

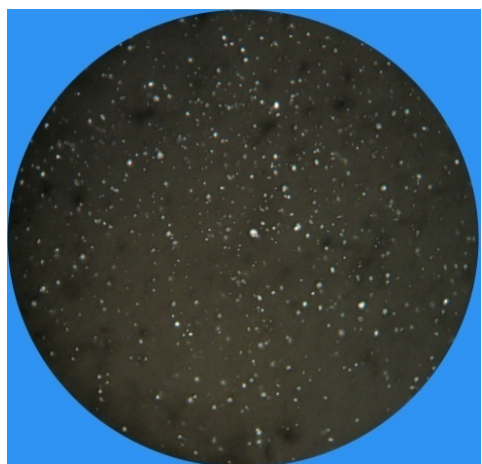

(e)

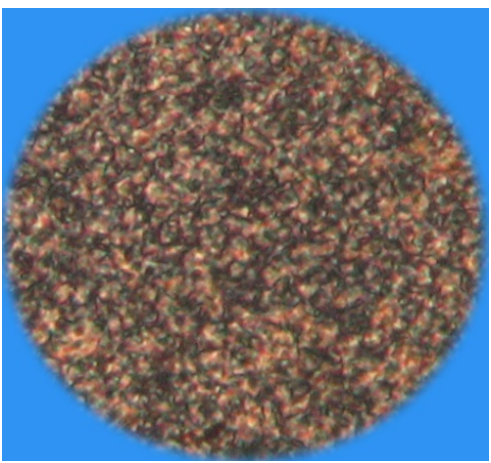

(b)

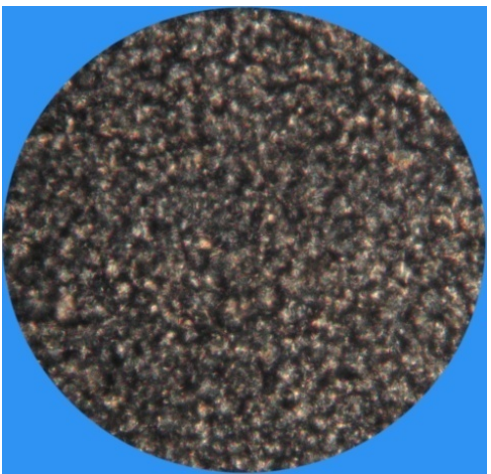

(d)

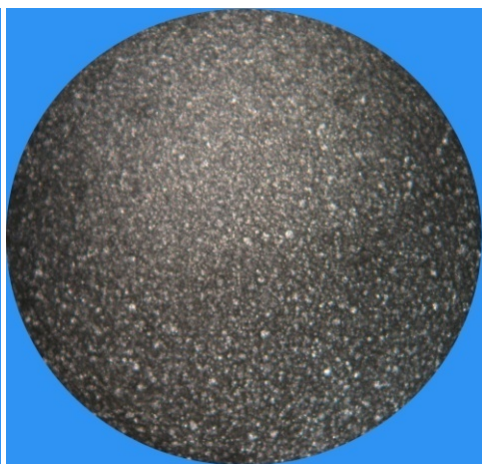

(f) 


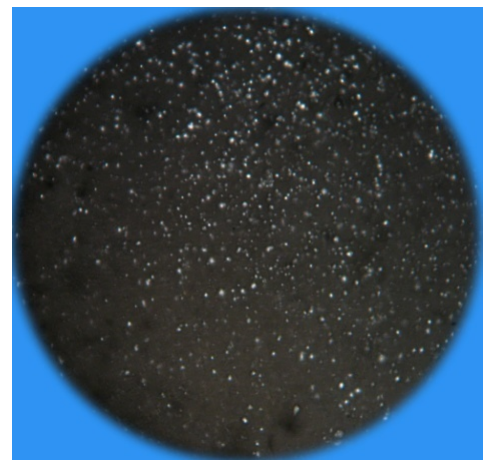

(g)

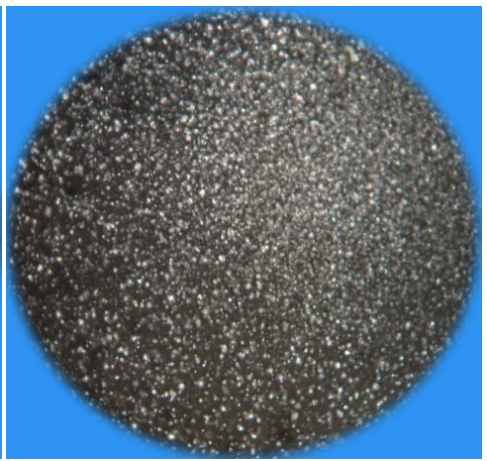

(h)

Figure 5. Crystallisation behaviour of developed polymers (a) $\mathrm{HDPE}$ at $130^{\circ} \mathrm{C}$; (b) $\mathrm{HDPE}$ at $120^{\circ} \mathrm{C}$; (c) $\mathrm{PP}$ at $130^{\circ} \mathrm{C}$; (d) PP at $120^{\circ} \mathrm{C}$; (e) $50 \mathrm{HDPE} / 50 \mathrm{PP}$ at $130^{\circ} \mathrm{C}$; (f) $50 \mathrm{HDPE} / 50 \mathrm{PP}$ at $120^{\circ} \mathrm{C}$; (g) $70 \mathrm{HDPE} / 30 \mathrm{PP}$ at $130^{\circ} \mathrm{C}$; (h) $70 \mathrm{HDPE} / 30 \mathrm{PP}$ at $120^{\circ} \mathrm{C}$.

\section{Conclusion}

The study concluded some salient features of the composite. The electrical properties like dielectric strength and volume resistivity are decreasing on reinforcement of PP to HDPE. On the other hand the surface resistivity is improved. The exact causes for the changes in the property are not clear. But it is believed maybe due to changes in the polarising property. The spherulite growth rate is higher for virgin PP than HDPE. The crystallization behaviour is broadly changed in the polymer blend. Co-occurring spherulites are seen in the polymer composite remarking the polyblend to be a physical mixture of two non compatible phases. The composite blend may find suitable application areas, if surface resistivity is under consideration.

\section{Conflicts of Interest}

The authors declare no conflicts of interest regarding the publication of this paper.

\section{References}

[1] Teroda, M. and Ohaba, Y. (1983) Energy Transfer Mechanism and Amplified Spontaneous Emission Characteristics of Dye Mixture Solutions. Journal of Applied Physics, 22, 1392-1396. https://doi.org/10.1143/JJAP.22.1392

[2] Gulrez, S.K.H., Ali Mohsin, M.E., Shaikh, H., Anis, A., Pulose, A.M., Yadav, M.K., Qua, E.H.P. and Al-Zahrani, S.M. (2014) A Review on Electrically Conductive Polypropylene and Polyethylene. Polymer Composites, 35, 900-914. https://doi.org/10.1002/pc.22734

[3] Walker, B.M. and Rader, C.P. (1979) Handbook of Thermoplastic Elastomers. Van Nostrand Reinhold, New York.

[4] Malik, T.M. and Prudhornme, R.E. (1984) Dielectric Properties of Poly( $\alpha$-Methyl $\alpha$-n-Propyl- $\beta$-Propiolactone)/Poly(Vinyl Chloride) Blends. Polymer Engineering \& Science, 24, 144-152. https://doi.org/10.1002/pen.760240212

[5] Pathrnanathan, K., Cavaille, J.Y. and Johari, G.P. (1988) Dielectric Relaxations of Microstructurally Different Latex Polymer Blends of Poly(Butyl Acrylate) and Poly(Vinyl Acetate). Polymer, 29, 311-319.

https://doi.org/10.1016/0032-3861(88)90339-4 
[6] Maistros, G.M., Block, H., Bucknall, C.B. and Patridge, I.K. (1992) Dielectric Monitoring of Phase Separation during Cure of Blends of Epoxy Resin with Carboxyl-Terminated Poly(Butadiene-Co-Acrylonitrile). Polymer, 33, 4470-4478. https://doi.org/10.1016/0032-3861(92)90402-I

[7] Pillai, P.K.C., Narula, G.R. and Tripathy, A.K. (1984) Dielectric Properties of Polypropylene/Polycarbonate Polyblends. Polymer Journal, 16, 575-578.

[8] Radhakrishnan, S. and Saini, D.R. (1994) Structure and Dielectric Properties of Poly(Vinyl Chloride) Thermoplastic Elastomer Blends. Journal of Applied Polymer Science, 52, 1577-1586. https://doi.org/10.1002/app.1994.070521106

[9] Gustafsson, A., Salot, R. and Gedde, U.W. (1993) Electrical Degradation of Blends and Laminar Composites of Polyethylene and Polystyrene. Polymer Composites, 14, 421-429. https://doi.org/10.1002/pc.750140509

[10] Mansour, A.A., Sabagh, S.E.L. and Yehia, A.A. (1994) Dielectric Investigation of SBR-NBR and CR-NBR Blends. Journal of Elastomers \& Plastics, 26, 367. https://doi.org/10.1177/009524439402600406

[11] Elashmawi, I.S., Hakeem, N.A. and Abdelrazek, E.M. (2008) Spectroscopic and Thermal Studies of PS/PVAc Blends. Physica B: Condensed Matter, 403, 3547-3552. https://doi.org/10.1016/j.physb.2008.05.024

[12] Ramesh, S., Yahaya, A.H. and Arof, A.K. (2002) Miscibility Studies of PVC Blends (PVC/PMMA and PVC/PEO) Based Polymer Electrolytes. Solid State Ionics, 148, 483-486. https://doi.org/10.1016/S0167-2738(02)00091-7

[13] Rawat, A., Mahavar, H.K., Tanwar, A. and Singh, P.J. (2014) Study of Electrical Properties of Polyvinylpyrrolidone/Polyacrylamide Blend Thin Films. Bulletin of Materials Science, 37, 273-279. https://doi.org/10.1007/s12034-014-0639-4

[14] Mothé, C., Monteiro, D. and Mothé, M. (2016) Dynamic Mechanical and Thermal Behavior Analysis of Composites Based on Polypropylene Recycled with Vegetal Leaves. Materials Sciences and Applications, 7, 349-357. https://doi.org/10.4236/msa.2016.77031

[15] Pillai, P.K.C., Gupta, A.K. and Goel, M. (1980) Study of Surface Potential Characteristics of Corona Charged Ethyl Cellulose Layers for Its Relevance in Electrothermography. Macromolecular Chemistry, 181, 951-956.

https://doi.org/10.1002/macp.1980.021810416

[16] Krishnakumar, B., Gupta, R.K., Forster, E.O. and Laghari, J.R. (1986) The Effect of Polypropylene Morphology on AC Breakdown. Annual Report Conference on Electrical Insulation and Electrical Insulation and Dielectric Phenomena, 522.

[17] Wagner, H. (1974) The Influence of Superstructures on the Electrical Breakdown of Partially Crystalline Polymers. Annual Report Conference on Electrical Insulation and Electrical Insulation and Dielectric Phenomena, 62.

[18] Kawahigashi, M., Miyashita, Y. and Kato, H. (1989) Chemical Structures of Copolymers and Their Electrical Properties. IEEJ EIM Study Meeting, EIM-89-50, 39. (In Japanese)

[19] Yamakita, T. (1990) Morphology and Dielectric Breakdown of Blend Polymers. IEEJ EIM Study Meeting, EIM-90-77, 1. (In Japanese)

[20] Katsunami, K., Ishii, K., Tanaka, Y. and Ohki, Y. (1991) Dielectric Properties of Polymer Blend of Polypropylene and Polyethylene. Proceedings of the 3 rd International Conference on Properties and Applications of Dielectric Materials, Tokyo, 8-12 July 1991, 999-1002. https://doi.org/10.1109/ICPADM.1991.172241

[21] Ku, C.C. and Leiens, R. (1987) Eleclrical Properties of Polymers: Chemical Principles. Hanser Publishers, New York. 